Castillejos Saucedo, Giovanna; González Machado, Nizaí; Ángel González, Karen Anahí del; Flores Pérez, Alfredo; Arroyo Urióstegui, Ana Julia; Correa Rodríguez, Ingrid; Flores Avalos, Martha; Mondragón Millán, Azucena; Barquera Mondragón Paula; Martí, Sandra Amelia.

Profesores, investigadores y alumnos de la Universidad Autónoma Metropolitana-Xochimilco.

\title{
Del objeto en transformación a la Investigación Artística.
}

\section{From the transformation object to the Artistic Research.}

\author{
TIPO DE TRABAJO: \\ Comunicación Virtual. \\ PALABRAS CLAVE: \\ Sistema Modular, Educación, Investigación, Collage, Práctica artistica, Arte, Diseño. \\ KEY WORDS:
}

Modular System, Education, Research, Collage, Artistic practice, Art, design.

RESUMEN.

El desarrollo de la Investigación Artística alcanza pertinencia para revalorar y abrir nuevas vias para la investigación y la relación enseñanza-aprendizaje para el diseño en la División de CyAD en la UAM-X en México. El binomio global-local representa una relación desde donde mirar la vías que como sujetos instituídos establecemos hacia prácticas instituyentes en la investigación y docencia. La figura de la coautoría es una primer resolutivo para este fin.

\section{ABSTRACT.}

The develpment of Artistic Research has become relevant to reevaluate and to open new paths to academic research and the relationship between education and learning in CyAD's -Cience and Arts for Design- Division in UAM-Xochimilco, Mexico. The binomial union bwteeen global and local notions represents a way of looking at how we as part of an institution establish institutional practices in investigation and teaching. The joint authorship in this paper is a first step towards this end.

\section{CONTENIDO.}

INTRODUCCIÓN

El objetivo de esta comunicación es mostrar las posibilidades de desarrollo de la Investigación Artística en la División de Ciencias y Artes para el Diseño (CYAD) de la Universidad Autónoma Metropolitana - Xochimilco, como resultado del curso-taller Teoría y técnica de la representación conceptual. Introducción a la investigación artística I. Posibilidades que pueden ser abordadas como contribuciones a las nociones de pedagogía colectiva, práctica cultural, autocritica del trabajo individual en el marco institucional -lo que podría derivar en una crítica institucional-, autogestión de aprendizajes, modos de producción y difusión del conocimiento. 
Este propósito se ubica en el marco de una coyuntura compuesta por los siguientes elementos: el Sistema Modular, modelo académico para la docencia e investigación en la División de CyAD, cuyo fundamento conceptual para la organización, producción y divulgación del conocimiento es el objeto de transformación; los numerosos artistas que conforman la planta docente cuya práctica investigativa está guiada por el modelo científico, aunado a los significados que las disciplinas Diseño y Arte comparten en la producción de la cultura visual.

El modo en que interesa integrar a la Investigación Artística es en cuanto a los modos, siempre en cambio y movimiento, que tiene para articular prácticas y saberes ya sea de diferentes disciplinas o de entornos en conflicto. Esto nos permitirá adentrarnos en la experiencia de instituir otras formas válidas de producir, organizar y divulgar el conocimiento y que a su vez, "nos permiten ver este mundo que encontramos y en el cual nos encontramos." (Xirau 1993, p. 5).

El binomio global-local se nos presenta como reflexión al final del curso. Hemos distinguido la tendencia global en la tradición académica, que preocupada por la preservación de sus estructuras se presenta con supremacía. Y lo local lo concebimos como la hendidura de la institución, donde nos ubicamos subjetivamente, desde la que es posible la incorporación de articulaciones antes no exploradas entre arte e investigación en la UAM $-\mathrm{X}$.

Por medio de la coautoría se quiere enfatizar que los participantes del curso, sus experiencias, saberes, prácticas y sus expectativas son el material con que el docente guía el contenido del curso. Se estimula la participación activa, constante y explícita de forma vital. La figura profesor-alumno se desborda y el conocimiento entra en una práctica indiferenciada en donde no hay una sólo fuente de información, en una dinámica de creación de espacios comunes y de colaboración. El docente -que hemos preferido llamar coordinador-, está específicamente encargado de hacer sobresalir el saber conceptual, vivencial y emocional de cada participante.

Esta comunicación se desarrolla en tres etapas, la primera muestra la integración de los fundamentos del Sistema Modular con diferentes posturas de la investigación artística. La segunda, muestra en la experiencia de la práctica artística que las relaciones teóricas antes desarrolladas son posibles. Y la tercera, el diagnostico que, a partir de preguntas, muestra el balance crítico que los participantes hacen de su experiencia en el curso bajo estos planteamientos.

\section{EL OBJETO DE TRANSFORMACIÓN Y LOS FUNDAMENTOS DE LA INVESTIGACIÓN ARTÍSTICA}

El Sistema Modular representa para nosotros una identidad académica y profesional, conocer sus fundamentos es acercarnos a las estructuras, nos demos cuenta o no, que nos conforman, incluso si estamos de acuerdo con ellas o no. Y el arte se nos presenta como una práctica o estudio alternativo, como algo que se realiza en los rato libres pero que tiene una constante incidencia en la vida dentro de la academia pero no se incorpora en plenitud como área problemática.

Esta división está fundamentada en el Sistema Modular pero organizada con base al Diseño como objeto de transformación. La estructura del Sistema Modular plantea la transformación de la realidad social, es decir, concibe la realidad en movimiento, en constante cambio y por lo tanto exige de la institución la capacidad de adaptación, reorganización y praxis. De ello, se desprende la noción de objeto de transformación, asocia de manera directa la relación conocer-transformar en un solo movimiento. Para la UAM-X “Conocer un objeto es conocer un acontecimiento, no es solamente mirarlo y hacer de él una copia mental, una imagen. Conocer un objeto es actuar sobre él. Conocer es modificar, transformar el objeto y entender el proceso de su transformación y, como una consecuencia, entender la forma en que el objeto es construido" (UAM-X Documento Xochimilco, 1994, citado en Arbesú UAM-X. p.14).

En el CyAD la noción de diseño comprende al diseño industrial, arquitectónico, gráfico y de planeación urbana, pero aún no es claro cuál es el objeto de transformación de la realidad, que a su vez, guía al diseño. Nótese que el nombre completo de la división deja clara la relación del diseño con las Ciencias y el Arte, pero ¿qué tipo de relación? Su relación con las Ciencias se asume más clara pues el fundamento del Sistema Modular se concibió desde las Ciencias Sociales y Biológicas que guiados por la investigación científica, se establecieron objetivos generales en común relacionados con "los problemas más importantes que enfrentaba la sociedad mexicana: salud, educación, producción agrícola e industrial, problemas financieros, políticos, sociales y otros" (Arbesú 2004, p.19). Por ello, la investigación para el diseño se sostiene con el modelo científico.

Entonces, ¿cómo se asume la naturaleza del fenómeno artístico para el diseño? Antes de eso, tómese en cuenta que el diseño no ha dejado de presentarse para los alumnos y docentes como una categoría problemática. Desde la conformación del Sistema Modular para el diseño fue complicado configurar cuáles eran los problemas que enfrentaba la sociedad mexicana en cuanto al diseño. A pesar de lo complicado de tal ejercicio, la división se organizó en cuatro procesos a los que corresponden cuatro departamentos: conceptualización (departamento de Teoría y Análisis), formalización (departamento de Síntesis creativa), materialización (departamento de Tecnología y producción) y aplicación de los diseños (departamento de Métodos y sistemas).

Sin embargo, la organización no remite a algún problema de la realidad. Esta ausencia no permite, desde nuestra consideración, distinguir la específica naturaleza del diseño para con la sociedad, la integración plena de las ciencias o de las artes cuya relación llega a 
Castillejos Saucedo, Giovanna; González Machado, Nizaí; Ángel González, Karen Anahí del; Flores Pérez, Alfredo; Arroyo Urióstegui, Ana Julia; Correa

Rodríguez, Ingrid; Flores Avalos, Martha; Mondragón Millán, Azucena; Barquera Mondragón Paula; Martí, Sandra Amelia

III CONGRESO INTERNACIONAL DE INVESTIGACIÓN EN ARTES VISUALES :: ANIAV 2017 :: GLOCAL [codificar, mediar, transformar, vivir] http://dx.doi.org/10.4995/ANIAV.2017.5835

ser más bien tangencial y reconocer la naturaleza específica del conocimiento que desarrolla en la investigación. Es en esta ausencia donde nace la opción de mirar en el arte, un desarrollo analógico que nos enseñe a mirar nuestra especificidad en el diseño.

Henk Borgdorf (2010) examina en el arte una vía de producción de conocimiento, afirma que la investigación artística además de develar la naturaleza del conocimiento que desarrolla, aporta articulaciones pre-conceptuales. Invita al pensamiento inacabado, conocimiento pensado en, a través y con el arte. Tal forma abierta del conocimiento no difiere de la noción de transformación por la que apuesta el sistema de la UAM, al contrario, se presentan análogamente como formas del saber que remiten, como dice Ramon Xirau cuando desarrolla la compatibilidad de la poesía con el conocimiento, "a cuestiones vitales que el hombre se plantea -nuestro origen, nuestro destino, el tiempo, la vida misma- [...] "problemas" humanos que son problemas de fundamento [...]" (Xirau 1993, p.4). Cuestiones que además no se desvinculan de las relaciones políticas, económicas, de comunicación, entre otras.

La naturaleza del lenguaje del arte contemporáneo -y aún en la poesía, naturaleza significada en imágenes-, apunta a una correspondencia entre experiencia, palabras, percepción y teoría, que como señala Chus Martínez "es ocasión para reformular el sentido de continuidad entre experiencia y lenguaje [...] la posibilidad de iniciar una obra de manera tal que ningún concepto esté en relación de exterioridad con ningún otro material, arte o ciencia, vivo o inerte, humano o no humano" (s/f). Esta naturaleza es nombrada como noconceptual, no porque no trabaje efectivamente con conceptos o en una dimensión lógica-abstracta, sino porque se espera, en todo caso un nivel de concreción vivo y múltiple que contribuya a definir relaciones sociales y culturales. Martínez aclara, "[...] no se trata sobre una polifonía. Esta otra forma, una proliferación dominante de estilos, maneras, lógicas, lenguajes, debe ser considerada como una que construye los materiales para convertirse en el locus de una actividad dialógica entre el arte y sus modos de indagar la naturaleza del conocimiento." (s/f)

\section{EL ALUMNO COMO SÍNTOMA DE LA REALIDAD}

Para exponer el proceso de práctica artística como reconocimiento del objeto de transformación configuramos la actividad como sigue:

En la primera sesión del curso, se elaboró un mapa colectivo (Fig.1), que reflejara mediante conceptos, los intereses que los participantes tenían en cuanto a la relación investigación-arte. Estos conceptos se encuentran en color rojo, y los hemos denominado "campos problemáticos". Los conceptos en color azul, rodean a los rojos y provienen de una interacción azarosa -como lluvia de ideas- que los participantes tuvieron con los campos problemáticos; sin embargo, precisemos que ese azar proviene de nuestros referentes culturales.

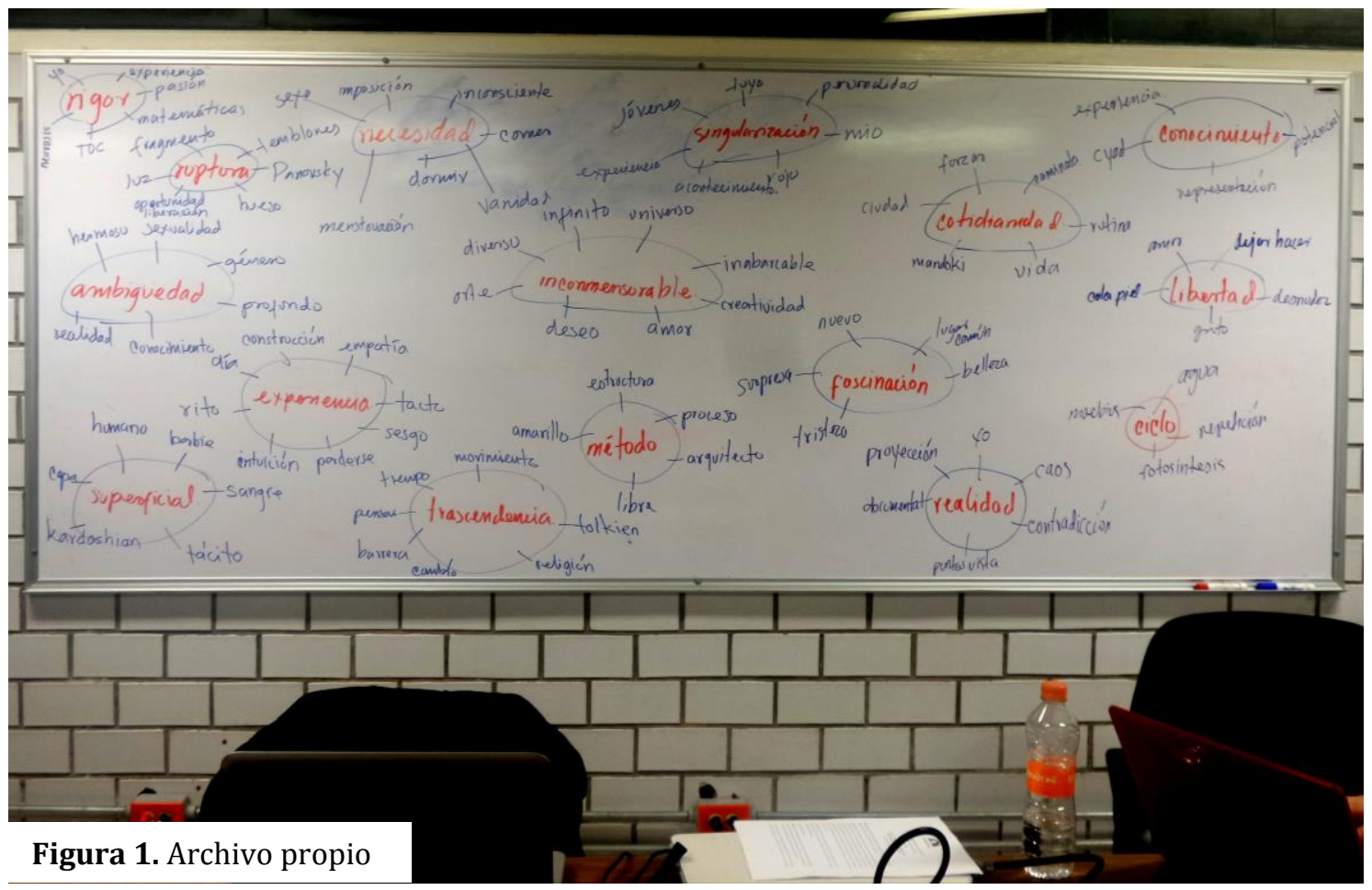

Cada participante estableció una ruta de acción, una selección de conceptos a manera de guion, a partir de cual vislumbrara un proceso de producción de collage (Fig. 2). Los conceptos se sustituyeron por analogía a un medio artístico, gráfica, audio, video, dibujo, etc. 
Castillejos Saucedo, Giovanna; González Machado, Nizaí; Ángel González, Karen Anahí del; Flores Pérez, Alfredo; Arroyo Urióstegui, Ana Julia; Correa Rodríguez, Ingrid; Flores Avalos, Martha; Mondragón Millán, Azucena; Barquera Mondragón Paula; Martí, Sandra Amelia

III CONGRESO INTERNACIONAL DE INVESTIGACIÓN EN ARTES VISUALES :: ANIAV 2017 :: GLOCAL [codificar, mediar, transformar, vivir] http://dx.doi.org/10.4995/ANIAV.2017.5835

Este ejercicio representa el trabajo conceptual en el que nos adentramos en el proceso de investigación: definición, selección, delimitación, relación y aporte conceptual a algún fenómeno. El objetivo de esta parte del ejercicio es activar en el participante dos condiciones: el condicionamiento adquirido que tenemos en el uso de las palabras y sus significados, reflejo de la idiosincrasia de cada quién, aunque la mayor de las veces compartida; sin embargo, es el bagaje con que un estudiante de maestría o licenciatura comienza a hacer investigación. La segunda condición es el leguaje no-conceptual, la imaginación, el deseo y el anhelo, que antes que aparecer como conceptos son la experiencia con el mundo y las cosas.

Dada esta selección los participantes definen los lineamientos de su propio problema de producción de collage, su metodología, las etapas en que se desarrollará y las perspectivas que vislumbran alcanzar. A partir de este punto, se fomenta la auto-observación, principio autocrítico de los modos de pensar y crear, a través de las preguntas: ¿por qué estoy relacionando "tal concepto" con "este otro"? ¿Qué cosa, realidad o problema conforman todas las relaciones establecidas? ¿Puedo nombrar todas las relaciones que estoy generando? ¿Puede ser conformada una unidad de sentido? Si no es posible, ¿por qué es importante que se mantenga la diferencia, es decir, que los fragmentos, aunque relacionados, mantengan cierta autonomía?

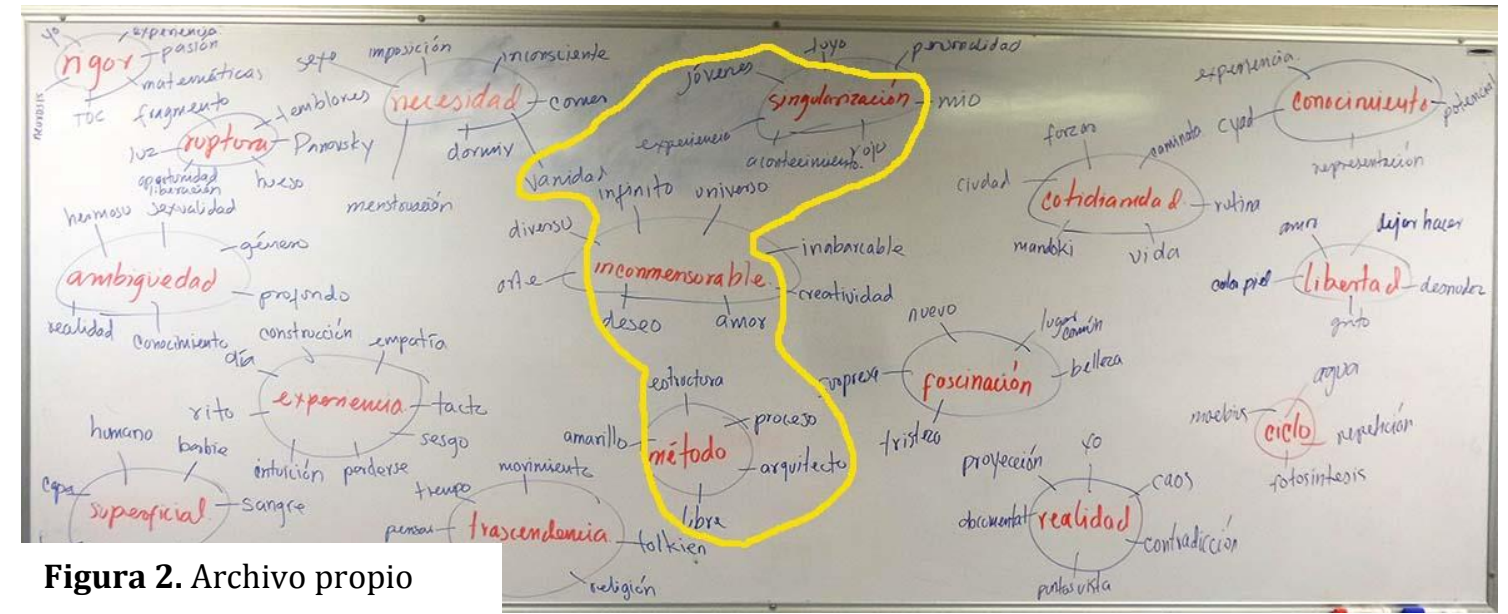

En adelante los procesos toman formas diferenciadas, a cada propuesta se le hacen recomendaciones y se fomenta que una mayor exigencia en el nivel de profundidad que su reflexión ha alcanzado. (Fig. 3 y Fig. 3bis)
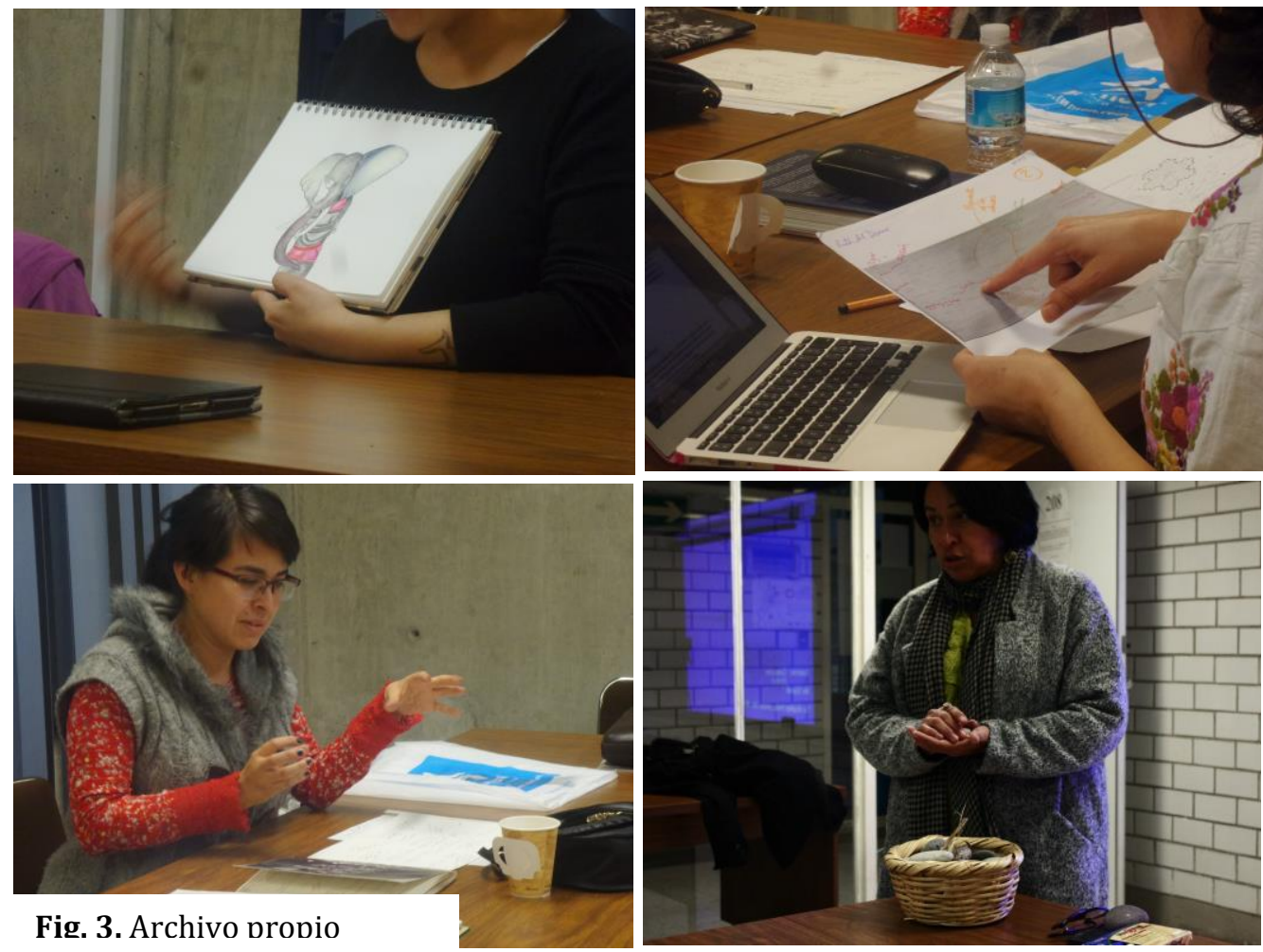

ANIAV Asociación Nacional de Investigación en Artes Visuales

This work is licensed under a Creative Commons Attribution-NonCommercial-NoDerivatives 4.0 International License (CC BY-NC-ND 4.0) 

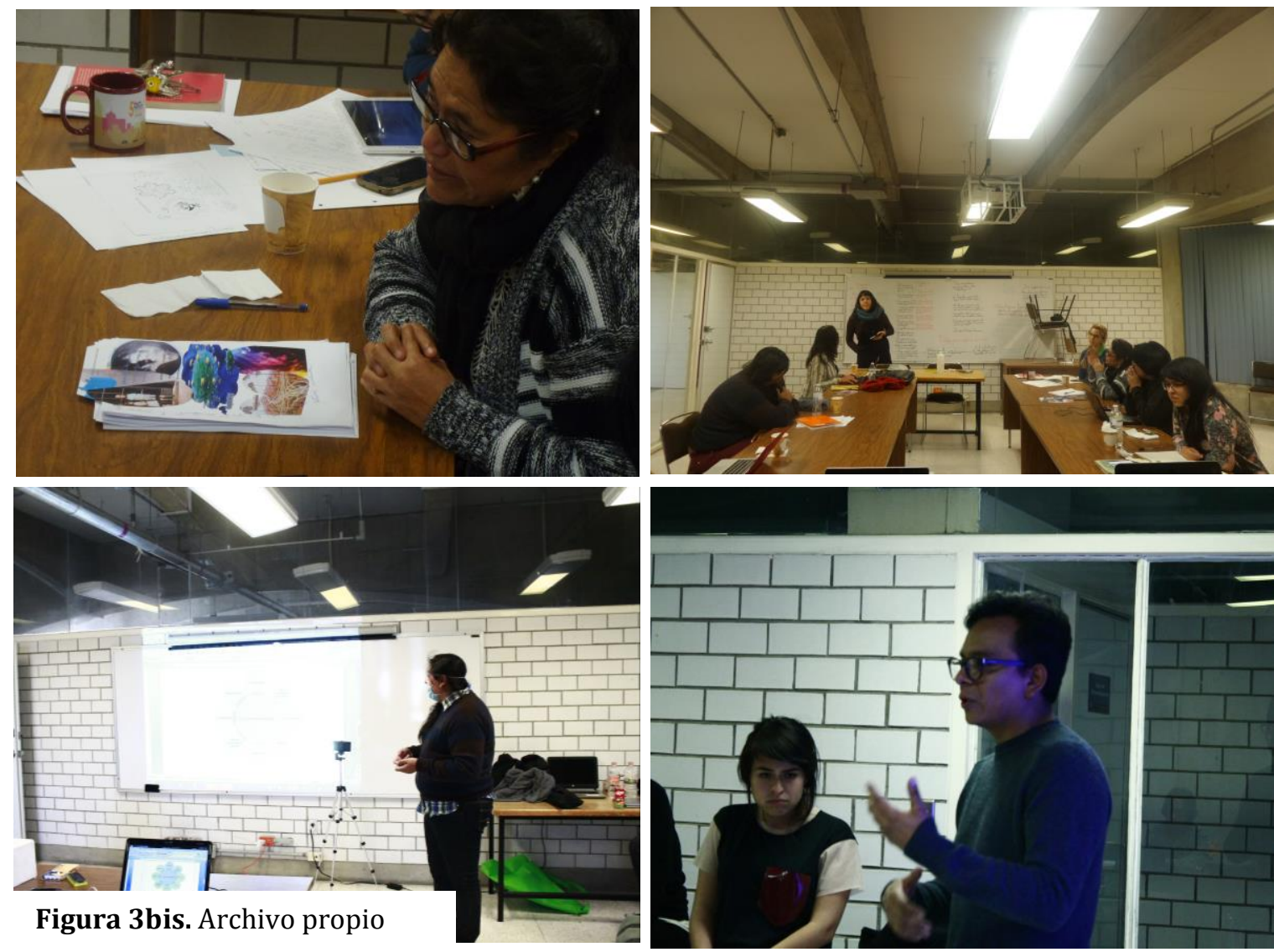

La última parte de este ejercicio consiste en redactar metodológicamente su propuesta, el proceso de cómo transcurrió el desarrollo y transformación de los conceptos; así como brindar su testimonio de si la práctica artística les permitió o no reconocer las relaciones culturales en el lenguaje y su posible modificación. En esta etapa fue importante enfatizar el darse cuenta de las formas personales con que organizamos nuestra producción, de los modos en que solemos acudir a nuestra experiencia, percepción u emoción para configurar un proyecto. La apropiación de estas reflexiones es lo que, consideramos, constituye la información de lo real, lo real como fenómeno para la investigación y producción a partir de la experiencia de las personas con el mundo.

\section{REPERCUSIONES DE LA PRÁCTICA ARTÍSTICA EN LA COMPRENSIÓN DEL OBJETO DE TRANSFORMACIÓN}

El ejercicio de collage como práctica artística del análisis conceptual nos permitió replantear el horizonte del quehacer investigativo en el CYAD. Nos acercamos a una noción amplia y consciente de las relaciones y procesos involucrados en el desarrollo de conocimiento dentro de la investigación, las artes y el diseño. El collage intensifica la idea de que la investigación, el arte y el diseño no pueden ser procesos lineales, sino actividades de carácter proyectual en que es necesaria la práctica de un pensamiento múltiple.

Para mostrar estos resultados entrevistamos a los integrantes del curso y registramos en video sus aportes. A continuación se presentan, en lo general, las nociones compartidas en la documentación.

¿Cómo se diferencia tu proceso de collage con los métodos y técnicas que empleas usualmente en tu trabajo de investigación?

Ajustados al protocolo de investigación, de que disponemos para proponer un trabajo de investigación, basado en el modelo científico; las prácticas responden a una guía estructural que da coherencia a las prácticas. En cambio, en el proceso de collage no-lineal los pasos no pueden ser mecánicos, en la realidad su desarrollo es más bien un proceder errático que toma un sentido propio conforme se va tomando consciencia de lo que se observa, en relación a las perspectivas individuales y a las revisiones teóricas. De modo que en la marcha lo que se desarrolla es un método propio en donde los conocimientos previos, los conocimientos nuevos y el deseo individual se encuentran coludidos. 
La experiencia consciente del trabajo analítico que se llevó a través de la práctica artística nos ofrece una suerte de claridad, en los modos en que cada uno hace investigación y que parte de preguntarse ¿por qué selecciono un tema específico para investigar?, responder esta pregunta conlleva a una auto-revisión profunda del cúmulo de experiencias personales que nos permiten comprender el punto de vista del que partimos y solo así, reconocer las posibilidades que se tienen para ofrecer; es decir que, somos tanto juez, como parte, de la acción de investigar. Esta claridad aporta también al quehacer docente, en el sentido que permite orientar mejor a los alumnos para que desarrollen métodos individuales que conduzcan a intervenciones profesionales significativas.

\section{¿Cómo cambia tu percepción del objeto de transformación?}

Emprender el collage como un proceso de investigación reafirma que la investigación a través de la práctica es el camino natural del trabajo en las artes y el diseño, un experiencia que rebasa y suple la experimentación material por la experimentación de lenguajes. Cuando el concepto pasa por el filtro de la práctica y recubre las necesidades del problema en transformación, se supera; de modo que después de la práctica hay un conocimiento que no había antes y otorga un sentido de continuidad que no se agota en la materialización de la representación discursiva -sea esta texto académico, obra de arte u objeto de diseño- sino que, genera un conocimiento que cobra vida propia y que continua transformándose en la realidad.

Desde nuestro campo, el esquema modular, cualquier problema de la realidad puede ser transformado, al llevar a la investigación un problema de interés individual el investigador se vuelve parte del problema mismo, por ello es que el primer objeto de transformación es el sujeto mismo y después, es la acción interdisciplinaria para diagnosticar un problema y, más que encontrar soluciones, dilucidar las diversas posibilidades de aportar en él.

El arte y el diseño reconocen que no hay una única fórmula de acción y permiten comprender todas las variables de transformación en el tiempo que se investiga. Los diversos lenguajes de las artes y el diseño pueden coparticipar en la investigación para desarrollar diagnósticos y llevar a la práctica situaciones de irrupción que quiebran visiones obsoletas, y que pueden constituir nuevos enfoques relevantes para transformar o comprender mejor los problemas de la realidad.

\section{¿Cómo aporta al proceso enseñanza-aprendizaje en la investigación para el diseño?}

Los lenguajes artísticos permiten mostrar cosas que desde los límites del ámbito académico no se podía; así también, nos lleva a la comprensión de que los diseños pueden ampliar sus horizontes cognitivos y por lo tanto su impacto en la realidad. En el hacer y conocer de la investigación y después de la experiencia práctica del collage, junto a las reflexiones teóricas de nuestros seminarios, consideramos necesario repensar epistemológicamente los diseños, los puntos mencionados pueden por momentos parecer obvios; sin embargo, son cosas que en la investigación se han descuidado: en el análisis de la otredad no se termina de permear en uno mismo; la investigación es también un proceso de llevar a la consciencia lo propio, revelarlo y materializarlo como una forma válida de conocimiento; al investigar hacemos traducciones de la realidad para compartir experiencias, conocimientos y generar nuevos, para este trabajo, el uso de los lenguajes no debería de limitarse, sino al contrario, deberían aprovecharse las posibilidades que ofrecen para para que los derivados de la investigación transformen con originalidad y relevancia nuestro entorno.

De tales reflexiones observamos que en nuestro hacer de investigación y docencia enfrentamos dos grandes retos, el primero de ellos, ¿cómo aterrizar la investigación artística en la realidad de la investigación académica? Para ello pensamos que es necesario modificar el objeto de transformación, que no sea solo un referente de los problemas de la realidad, sino que, la acción de investigar en él, le otorgue cuerpo y lo convierta en un verdadero fenómeno de transformación.

Por otro lado, consideramos que la experiencia artística debería fluir naturalmente en la práctica investigativa, ese es el otro reto, asir los procesos de la investigación, las artes y el diseño y articularlos para que se permita el diálogo entre ellos. Trascender a las estructuras de las investigaciones académicas porque cada vez es más evidente que el sesgo debilita sus posibilidades de contribuir a una realidad que se encuentra en urgencia de cambio.

\section{¿Cuál es la importancia de la inserción practica en el desarrollo teórico investigativo?}

Somos conscientes de que la importancia de la investigación artística en relación con lo académico, es la reflexión y la socialización que se ejerce mientras se hace investigación, que aporta reconocimiento y libertad de los diversos saberes y formas de conocer que construyen una realidad, que impulsan el descubrimiento de uno mismo en un contexto específico lo que constituye un enfoque nuevo para observar y contribuir en la realidad. La investigación artística aporta esa originalidad en las contribuciones del diseño a la investigación y hace posible el brinco a lo académico; en este sentido, el arte y el diseño pueden contribuir a la construcción de conocimiento sin importar si se cuenta o no, con la validez institucional; sin embargo, sí tenerla, contribuiría a una mayor producción de aportes a través de la investigación. 
Castillejos Saucedo, Giovanna; González Machado, Nizaí; Ángel González, Karen Anahí del; Flores Pérez, Alfredo; Arroyo Urióstegui, Ana Julia; Correa

Rodríguez, Ingrid; Flores Avalos, Martha; Mondragón Millán, Azucena; Barquera Mondragón Paula; Martí, Sandra Amelia

III CONGRESO INTERNACIONAL DE INVESTIGACIÓN EN ARTES VISUALES :: ANIAV 2017 :: GLOCAL [codificar, mediar, transformar, vivir]

http://dx.doi.org/10.4995/ANIAV.2017.5835

Por ello es indispensable que dejemos de limitar los proyectos de investigación en el CyAD, dejar de maquillarles todo rastro puro de arte y diseño para que puedan ser aceptados por los criterios académicos, confrontar al sistema con la convicción clara de que no se pueden hacer descubrimientos de la vida si nos encontramos lejos de ella; entonces, que investigar a través de la práctica es hacer una conexión directa con lo vital, es una relación con la vida misma, con las experiencias propias, con el mundo y con uno mismo; de tal manera que el trabajo de la academia es el de determinar la viabilidad de los proyectos, pero también el de transformar la realidad y la teoría con ella, el de replantearla y si es necesario, destruirla.

Dicen los zapatistas que las preguntas sirven para caminar, y si nuestro fin es conocer-transformar, no es posible quedarnos quietos y amarrados, teoría-práctica-práctica teoría, un proceso sin fin.

\section{CONCLUSIONES}

Basados en la experiencia como estudiante, profesor y ahora investigador, nos damos cuenta que, a pesar de los grados de formación que vamos alcanzando, a pesar de las diferentes dinámicas aprendidas, siempre hay algo mejor que decir. Esa voz, no sólo compete a la figura de la autoridad, la figura de callar para escuchar y escuchar para aprehender. La guía de docencia y enseñanza proviene de la escucha mutua, una escucha que también comprende de diferencias y de grados de desarrollo. Por ello, consideramos que esta experiencia nos permite entrar en un nuevo campo de comprensión: la pedagogía colectiva.

Como práctica cultural nos ha permitido llevar a cabo una relación de acompañamiento, en un entorno en donde la palabra arte se dice quedo o es una palabra sorda que defiende la tradición. Nos ha permitido, además, un reconocimiento de otros valores que nos acompañan, las palabras inconmensurable, método y trascendencia se volvieron palabras libres y cargadas de sentido. La investigación se os presenta fuera de los sistemas y deviene un ejercicio cotidiano al que imprimimos rigor, estudio y esfuerzo para socializarlo y divulgarlo.

Hemos comprendido que los conceptos no son cajas contenedoras, son procesos en construcción en que nos involucramos $Y$ reconocemos autocríticamente, en una experiencia de interiorización y exteriorización procesual de los elementos del mundo que se nos presentan como particulares y como universales. Así mismo, este proceso de reconocimiento propio nos ha permitido vías de diálogo institucional, de alternativas de trabajo y relación, de forcejeo y negociación con los modos y bases educativas del sistema en el que nos encontramos.

\section{FUENTES REFERENCIALES.}

ARBESÚ, I.; BERRUECOS L. 1996. El Sistema Modular en la Universidad Autónoma Metropolitana. México: UAM-X.

BORGDORFF, H. 2005. El debate sobre la investigación artística. En Kunst als OnderzoeK. 6 de febrero 2004. Disponible en:http://www.ahk.nl/lectoraten/onderzoek/

FUENTES, C.E. 2003. Lecturas básicas I. Sistema Modular, la UAM-X y la Universidad Pública. México: UAM-X.

GARCÍA, S.S.; BELEM, P. S.

2011. Perspectivas ontológicas, epistemológicas y metodológicas de la investigación artística. Paradigmas, (3), pp. 89107.

KARLSSON, H.; BIGGS, M. (eds.). October, 2010. The Production of knowledge in Artistic Research. The Routledge Companion to Research in the Arts. Amsterdam School of the Arts, Royal Academy of Fine Arts, Music and Dance, The Hague.

LÓPEZ CUENCA, A. 2012. Acerca de la epistemología desboordada: conocimiento, prácticas artísticas y capitalismo cognitivo. En El incesante cicloentre idea y acción (págs. 90-99). México: Instituto Nacional de Bellas Artes.

MALO DE MOLINA, M. 2004. Nociones Comunes, parte 2: del análisis institucional a experiencias contemporáneas entre investigación y militancia, enTranversal. instituient practices. European Institiue for Progressive Cutlural Policies. Disponible en: http://eipcp.net/transversal/0707/malo/es

MARTÍNEZ, C. (s/f). Cómo un renacuajo se convierte en sapo. Estética tardía, política y materia animada: hacia una teoría de la investigación artística.Disponible en: https://es.scribd.com/document/328329772/Martinez-Chus-Como-UnRenacuajo-Se-Convierte- en-Sapo. 
Castillejos Saucedo, Giovanna; González Machado, Nizaí; Ángel González, Karen Anahí del; Flores Pérez, Alfredo; Arroyo Urióstegui, Ana Julia; Correa Rodríguez, Ingrid; Flores Avalos, Martha; Mondragón Millán, Azucena; Barquera Mondragón Paula; Martí, Sandra Amelia

III CONGRESO INTERNACIONAL DE INVESTIGACIÓN EN ARTES VISUALES :: ANIAV 2017 :: GLOCAL [codificar, mediar, transformar, vivir] http://dx.doi.org/10.4995/ANIAV.2017.5835

UNIVERSIDAD AUTÓNOMA METROPOLITANA. 1992. El proyecto académico de la Universidad Autónoma

Metropolitana Xochimilco. México.

XIRAU, R. 1993. Poesía y Conocimiento. Dos Poetas y lo Sagrado. México: el Colegio Nacional. 